\title{
Does Ferrule's Presence Affect the Success Rate of Bonded Restorations? A Systematic Review
}

\author{
Behnoosh Jalalian ${ }^{1}$, Ebrahim Abbasi ${ }^{2}$, Zahra Jafarian $^{3}$
}

\begin{abstract}
Aim and objective: Ferrules can reinforce the endodontically treated teeth by encircling the remaining coronal structure. The adhesive connection of bonded restorations to dental dentine via resin bonds on one hand and the importance of conservative tooth structure removal during crown preparations present the question "is ferrule necessary for bonded restorations?". This systematic review aimed to study the available literature on the effect of the ferrule on resin-bonded restored teeth.

Materials and methods: A search of the literature in PubMed and PMC's databases, without any date restriction, was carried out. The keywords were based on the PICO question "Does ferrule's presence affect the success rate of bonded restorations?". The full title and abstract of each article were screened by two independent authors using predetermined inclusion and exclusion criteria.

Results: Ten articles were included in this review. All teeth were endodontically treated and received lithium disilicate ceramic crowns bonded adhesively. Ferrules designs ranged from no ferrule to $3 \mathrm{~mm}$ and/or different ferrule circumferences. Three studies reported a lack of a significant difference in fracture resistance and/or stress levels and/or cyclic fatigue between different ferrule designs. Six studies reported higher fracture resistance, lower stress levels on root dentin and fiberglass post, higher count of fatigue cycles, less fracture count, less tooth strain value for ferrule groups.

Conclusion: In the limits of this systematic review, a ferrule is still necessary for resin-bonded restorations, and more ferrule in terms of height or/and circumference presents better results than no ferrule at all or interrupted or less ferrule.

Keywords: Bonded restoration, Ferrule, Systematic review.

International Journal of Prosthodontics and Restorative Dentistry (2021): 10.5005/jp-journals-10019-1332
\end{abstract}

\section{INTRODUCTION}

Endodontically treated teeth with severe loss of coronal structure propose a challenge for restorative dentistry and there is still a controversy over the choice of ideal treatment for such teeth. ${ }^{1}$ Endodontically treated teeth may face severe tooth structure loss due to caries, preexisting restorations, or the endodontic treatment procedure itself, which leads to an increased chance of fracture in function. ${ }^{2,3}$

Root-filled teeth restoration success requires an effective coronal seal, remaining tooth protection, functional restoration, and esthetics. ${ }^{4}$ Such teeth restoration may be achieved using direct and indirect restorations. The indirect full coverage method is the preferred one among clinicians. ${ }^{5} \mathrm{~A}$ post retained crown may be used to restore such teeth which could fail due to root fracture. Crown and post preparation designs aiming at the reduction of this phenomenon's chance are advantageous. Post insertion does not strengthen the endodontically treated tooth but it provides proper retention for the core material. ${ }^{6,7}$ If the coronal hard tissue has been lost severely, a post and core procedure may be required, which can increase the root perforation risk and may increase tooth fracture risk due to the additional dentine removal required for the preparation., ${ }^{1,8}$

The band of metal encircling the coronal surface of the tooth is called a dental ferrule, which is proposed to be effective in the reinforcement of root-filled teeth. The ferrule effect is the strengthening of the tooth due to the ferrule's resistance to stresses such as functional lever forces, the wedging effect of tapered posts, and the lateral forces exerted after post insertion. ${ }^{9}$ The ferrule can reinforce the endodontically treated tooth by encircling the remaining coronal structure leading to resistance against the functional lever forces during mastication. ${ }^{10}$

\begin{abstract}
${ }^{1}$ Dental Research Center, Dentistry Research Institute, Department of Restorative Dentistry, School of Dentistry, Tehran University of Medical Science, Tehran, Islamic Republic of Iran

${ }^{2}$ School of Dentistry, Qom University of Medical Science, Qom, Islamic Republic of Iran

${ }^{3}$ Department of Prosthodontics, Qom University of Medical Science, Qom, Islamic Republic of Iran

Corresponding Author: Zahra Jafarian, Department of Prosthodontics, Qom University of Medical Science, Qom, Islamic Republic of Iran, Phone: +2177486526, e-mail: iddzj1988@gmail.com
\end{abstract}

How to cite this article: Jalalian B, Abbasi E, Jafarian Z. Does Ferrule's Presence Affect the Success Rate of Bonded Restorations? A Systematic Review. Int J Prosthodont Restor Dent 2021;11(3):125-131.

Source of support: Nil

Conflict of interest: None

The endocrown is an indirect treatment option described as a full-coverage restoration with a circumferential butt-joint margin and a central retentive feature that extends into the pulp chamber space. ${ }^{11}$ Computer-aided design/computer-assisted manufacturing (CAD/CAM) has suggested that the endocrown restoration's adhesive technology is more conservative than post and core procedures. ${ }^{12}$

The resin-bonded restoration was introduced in the 1970s. ${ }^{13}$ Their retention is based on the wrap-around design and the bond to etched enamel. ${ }^{14}$ The main advantages of resin-bonded restoration are preserving tooth structure, reduction of pulpal morbidity, and better esthetics. This is especially true when no more aggressive treatment modalities can be born, because of age, medical 
condition, or finances. ${ }^{15}$ One of the materials used in bonded restorations is lithium disilicate. It has excellent biocompatibility, mechanical strength, and very good esthetic features. ${ }^{16}$ Moreover, it has a high strength of adhesion to the substrate due to both micromechanical and chemical bonding mechanisms. ${ }^{17}$

Magne et al. ${ }^{1}$ studied teeth with no ferrule restored with resin-bonded restorations and reported a high survival rate under fatigue tests. In another study, molars without ferrule were restored using bonded glass-ceramic crowns and showed that such teeth withstood high counts of cyclic loads. ${ }^{18}$ The resin bond quality of resin-bonded restoration poses the question "whether classic ferrule concepts still are necessary for these restorations or not?". Although fracture resistance increasing due to the ferrule has been previously studied. ${ }^{19}$ But the effects of ferrule features on resin-bonded restoration have not been systematically reviewed before. Therefore, this systematic review aimed to study the available literature on the effect of the ferrule on resin-bonded restored teeth.

\section{Materials and Methods}

\section{Focused Question}

The present study is a systematic review that was performed following the "Preferred Reporting Items for Systematic Review and Meta-Analysis Protocols" (PRISMA-P, 2015). ${ }^{20}$ The search was performed using "MeSH" terms and keywords based on the elements of the PICOS question which are presented in Table 1.

\section{Inclusion and Exclusion Criteria}

Inclusion Criteria

- In vitro studies.

- Without date restriction until February 15, 2021.

- Bonded restoration of any type of studies including but not limited to onlys, overlays, and endocrowns.

- With full-text availability.

- Written in English.

- Published in PubMed or PMC cited scientific journals.

\section{Exclusion Criteria}

- Articles about bonded restorations without any comparison between different ferrule heights.

- Review, in vivo, human, and animal studies.

- Written in a language other than English.

\section{Search Strategy}

An exhaustive search of the literature available in PubMed and PMC's electronic databases, without any date restriction until

Table 1: PICOS question elements

\begin{tabular}{ll}
\hline \multicolumn{2}{c}{ PICOS question } \\
\hline Participants $(\mathrm{P})$ & Teeth in need of restorations \\
Intervention $(\mathrm{I})$ & $\begin{array}{l}\text { Restoration using bonded } \\
\text { restorations }\end{array}$ \\
Comparison or control (C) & Presence or lack of ferrule \\
Outcome measures $(\mathrm{O})$ & Laboratory evaluations of \\
& fracture resistance, failure stress, \\
& failure modes, fracture counts, \\
& fatigue cycles, etc. \\
Types of studies included $(\mathrm{S})$ & In vitro studies \\
\hline
\end{tabular}

February 15, 2021, was carried out. The following keywords were used in the search strategy: (()(((()nlay) OR overlay) OR coverage) OR endocrown) OR coverage) OR crown) OR bridge) AND ((((glass ceramic) OR Emax) OR e.max) OR bonded ceramic)) AND ((ferrule) AND ferrule effect) AND ((()((clinical evaluation) OR clinical trial) OR longevity) OR success) OR failure) OR survival rate). The database search resulted in a total of 203 studies, 3 duplicates were found. The full title and abstract of each article were screened by two independent authors using predetermined inclusion and exclusion criteria.

\section{Study Selection}

Fourteen articles were chosen for full-text assessment from which eight were selected to compose this systematic review. The articles' initial screening was carried out using the Rayyan QCRI13 systematic review web app. ${ }^{21}$ All the references were selected from the Endnote program (Thomson Reuters, Philadelphia, PA, USA). To ascertain whether any relevant studies have been neglected in the initial search, references from the selected studies were also checked. Two more studies were included based on this hand search. Any differences in the selection of eligible studies were resolved by discussion with a third reviewer.

\section{Data Extraction}

$\beta$ Data were extracted from the included articles by two independent authors. A third author resolved any disagreements in the extracted data. Microsoft Office Excel software (Microsoft Corporation, Redmond, WA, USA) was used for the standardization and organization of the extracted data. The following information was collected:

- General characteristics of the selected studies: title of the article, first author, year, type of study, the title of the journal in which it was published.

- Tooth source and type and quantity.

- Root canal therapy information.

- Ferrule design and height.

- Crown, bond, and post and core material if present.

- Discoveries include fracture, failure, fatigue analysis (Table 2).

\section{Risk of Bias and Quality Assessment}

The risk of bias assessment method for the included articles was adapted from previous systematic reviews on in vitro studies. ${ }^{22,23}$ The following criteria were used to rate the articles:

- Selection of study groups: sample size calculation, description of clear inclusion/exclusion criteria, a detailed protocol of data collection.

- Detail of treatments on each group including post and core, buildup, ferrule design, bonding.

- Comparability: comparability of patients based on the study design or analysis and management of potential confounders.

- Outcomes: evaluation of results, assessment of accuracy outcomes.

- Statistical analysis: appropriateness/validity of statistical analysis and unit of the analysis reported in the statistical model.

Twenty points could be given to each study; $15-20$ points indicated high methodological quality, 10-15 points indicated medium-level, and others were considered as studies with low methodological quality. Two independent authors implemented this assessment. 


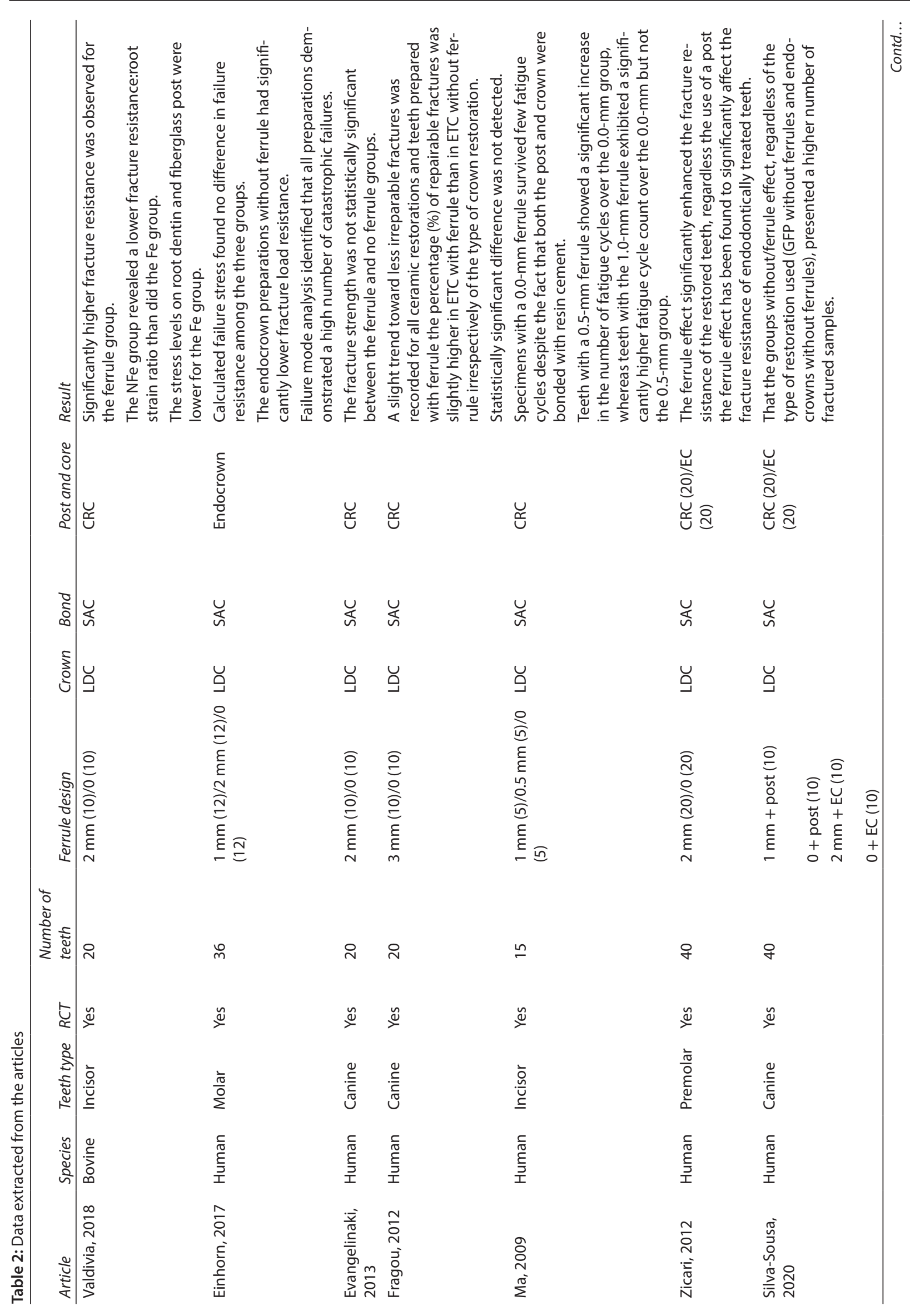




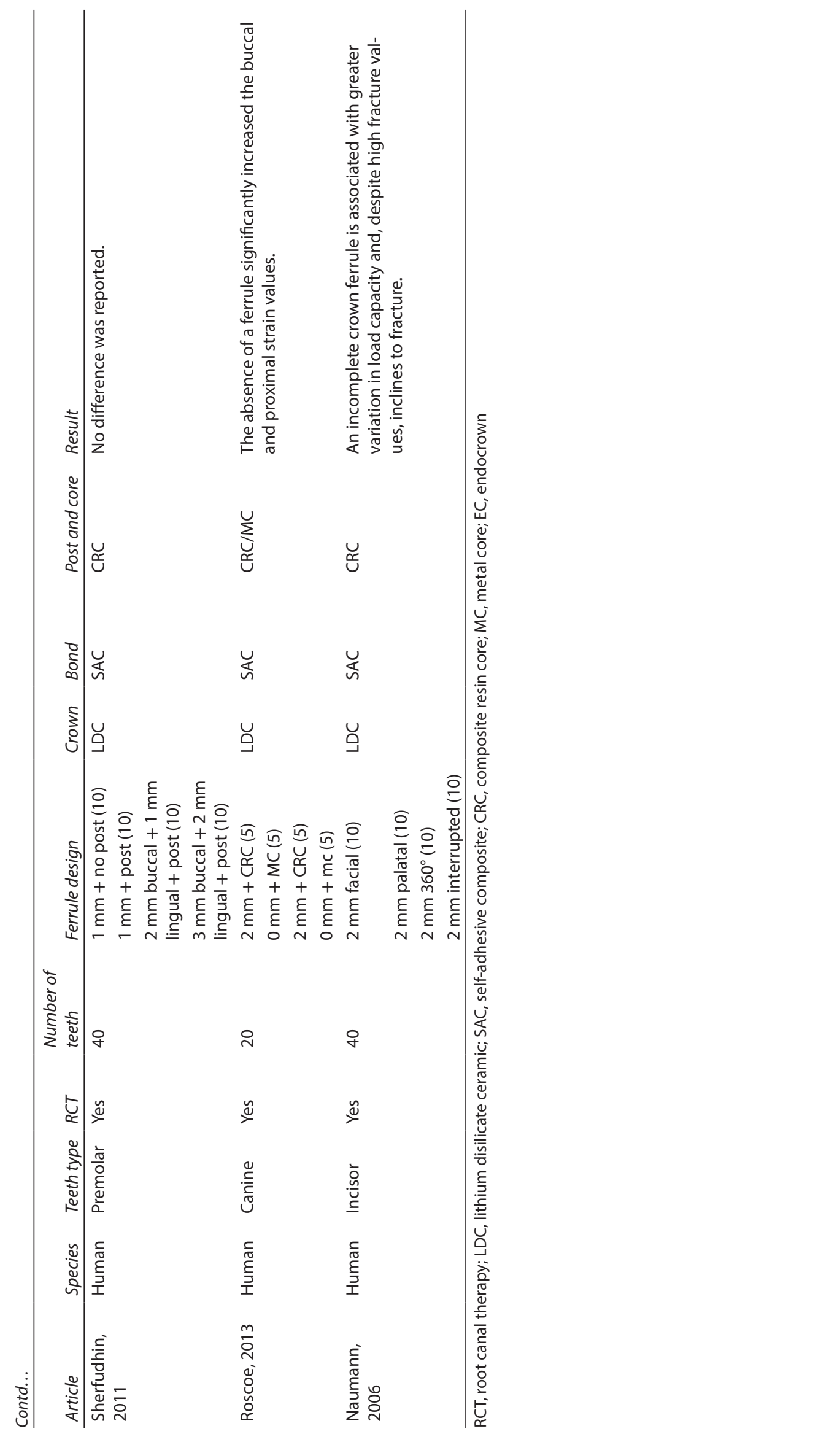




\section{Results}

\section{Literature Search}

A total of 203 studies were in the database search. Three were duplicates, and 186 were excluded after reviewing the titles and abstracts; 14 articles were chosen for full-text assessment. Four articles were excluded due to the lack of any comparison between different ferrule designs. Two other studies were excluded because they were review articles. Two studies were added to the review manually by going through the included articles' references thoroughly. In the last step, a total of 10 articles were included in the qualitative assessment (all in vitro studies, 9 on human teeth and 1 on bovine teeth) (Flowchart 1). The extracted data from included articles are presented in Table 2.

\section{Quality Assessment}

All but one of the studies included in this review received 15-20 points out of the possible 20 points, which is of high-level methodological quality (Fig. 1).

\section{Study Description}

Two hundred and ninety-one teeth were studied among the studies. Incisors (75), canines (100), premolars (80), and molars (36) were studied. All teeth were endodotically treated and received lithium disilicate ceramic crowns bonded adhesively. Two hundred and five of the teeth received composite resin posts and cores, 76 teeth received endocrowns without any posts, and 10 teeth received metal cast cores.

Different ferrules were designed in the included studies ranging from no ferrule to $3 \mathrm{~mm}$ ferrules. Two studies compared different ferrule circumferences (only buccal, only palatal, interrupted, and $360^{\circ}$ ferrules). ${ }^{24,25}$

Three studies ${ }^{25-27}$ reported a lack of a significant difference in fracture resistance and/or stress levels and/or cyclic fatigue between different ferrule designs. Two of them compared ferrule vs no ferrule, ${ }^{26,27}$ and the other used different ferrule circumferences. ${ }^{25}$

Six studies reported higher fracture resistance, ${ }^{24,28-30}$ lower stress levels on root dentin and fiberglass post, ${ }^{28}$ higher count of

Flowchart 1: Flow diagram of literature search and selection criteria adapted from PRISMA
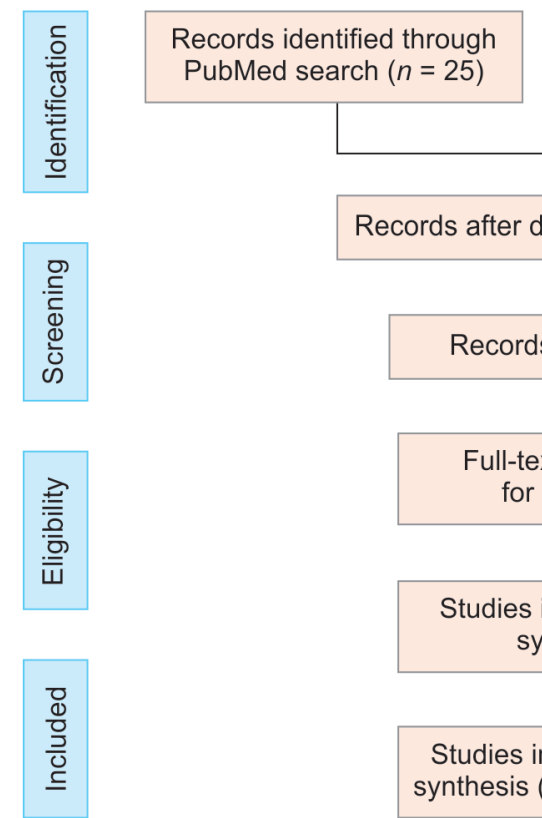

Additional records identified through PubMed central $(n=178)$

Records after duplicates removed $(n=200)$

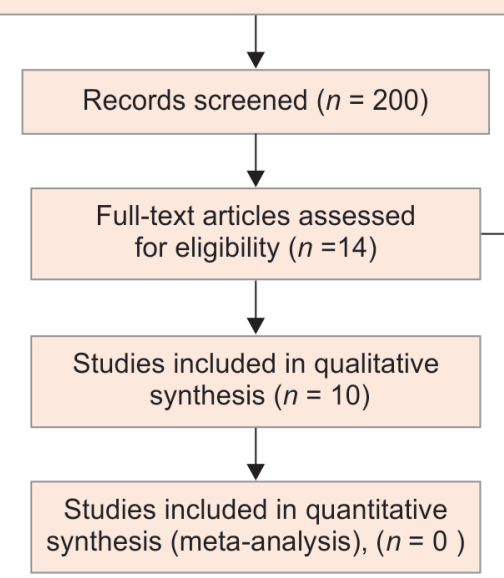

Full-text articles excluded $(n=6)$. Reasons

1. Review article $(n=2)$

2. No Ferrule's comparison $(n=4)$

Full-text articles included based on hand search $(n=2)$

synthesis (meta-analysis), $(n=0)$

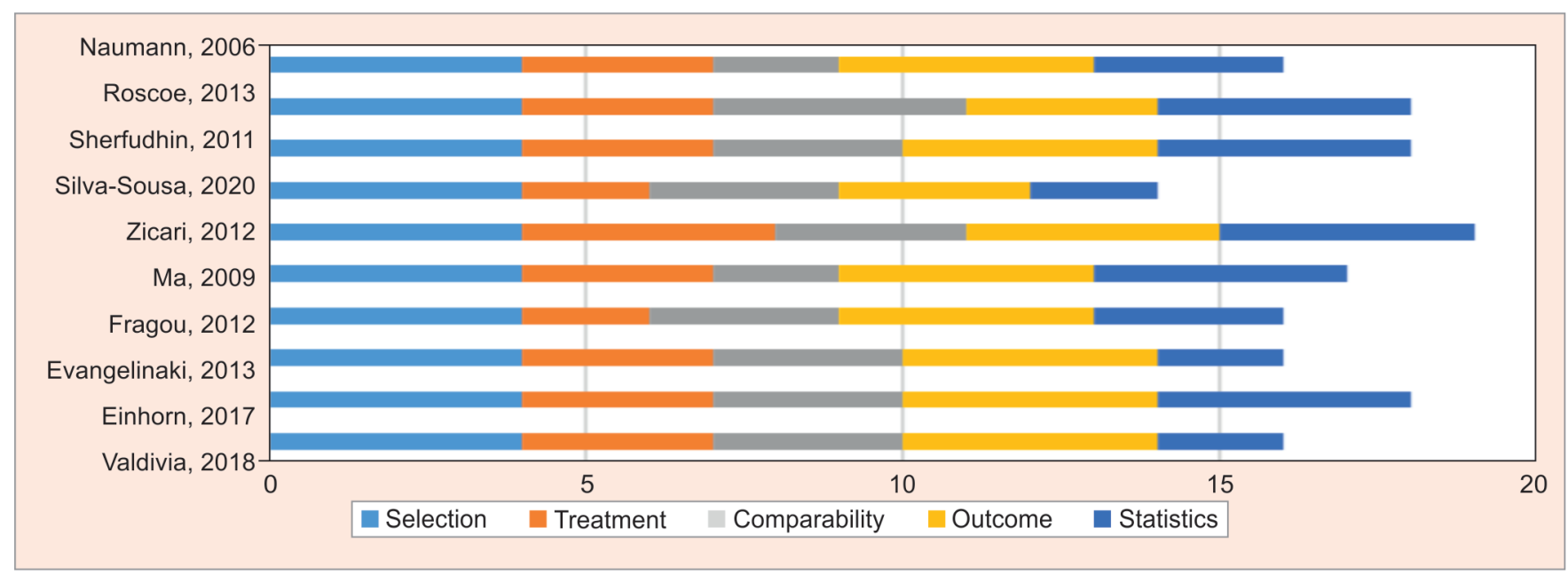

Fig. 1: Validity of studies based on selection criteria, treatment detail, comparability, outcome, and statistics 
fatigue cycles, ${ }^{31}$ less fracture count, ${ }^{32}$ less tooth strain value ${ }^{33}$ for ferrule groups. Naumann et al. ${ }^{24}$ compared ferrules with different circumferences (palatal, facial, $360^{\circ}$, and interrupted), and reported that incomplete crown ferrule is associated with greater variation in load capacity and inclination to fracture.

\section{Discussion}

The adhesive connection of bonded restorations to dental dentine via resin bonds on one hand and the importance of conservative tooth structure removal during crown preparations present the question "is ferrule necessary for bonded restorations?". Therefore, this systematic review aimed to investigate the effect of different ferrule designs on the success of teeth restored with bonded restorations. A meta-analysis was deemed inappropriate due to many affecting factors leading to incomparable results among studies. Factors such as different tooth sources and types, different experimental methods, different ferrule designs. Some studies have suggested a direct influence of ferrule on the success rate of endodontically treated teeth, ${ }^{34}$ but some others have found no effect of different ferrule heights for teeth treated with fiber posts and resin cores. ${ }^{35,36}$

The difference among the species from which the teeth were obtained for the included study could have affected the results of the study but even in the only study which used bovine incisor teeth, the results were similar to other studies, and significantly higher fracture resistance was observed for the ferrule group, the 0 ferrule group revealed a lower fracture resistance: root strain ratio than did the ferrule group, and the stress levels on root dentin and fiberglass post were lower for the ferrule group. ${ }^{28}$

Different types of teeth were studied in the included articles. Different anatomy of the studied teeth could result in different conclusions which could stem from different thicknesses of dentine or length of the root. Studies with no significant difference between ferrule designs ${ }^{25-27}$ as well as studies with significant differences ${ }^{30,32,33}$ included canine and premolars, therefore no conclusion could be made on the effects of tooth type on how ferrule could affect bonded restorations, can be made from the included studies.

Only one study used metal cast posts, ${ }^{33}$ but the results for both metal cast posts and composite resin cores were the same in this study, the absence of a ferrule significantly increased the buccal and proximal strain values.

One study used endocrowns, ${ }^{29}$ and two studies used both composite resin cores and endocrown (compared different ferrule designs in each group separately). ${ }^{30,32}$ The results for endocrowns remain the same, the endocrown preparations without ferrule had significantly lower fracture load resistance and the ferrule effect was found to significantly affect the fracture resistance of endodontically treated teeth. It can be deducted that ferrule shows better results than no ferrule regardless of post type and presence of a post.

Fragou et al. ${ }^{26}$ despite reporting a lack of significant difference between $3 \mathrm{~mm}$ of the ferrule and no ferrule in the tendency toward the type of failure, reported a slight trend toward less irreparable fractures for all-ceramic restorations and that in teeth prepared with ferrule the percentage of repairable fractures was slightly higher in ferrule groups.

Although both significant and not significant differences in fracture resistance, stress levels on root dentin and fiberglass post, count of fatigue cycles, fracture count, and tooth strain value have been reported, even in studies that failed to report a significant difference, the ferrule group showed better results. ${ }^{25-27}$ Although different heights of the ferrule and different circumferential designs of ferrule have been studied, taller ferrules and complete circumferential ferrules tend to show better results.

\section{Conclusion}

In the limits of this systematic review, a ferrule is still necessary for resin-bonded restorations. More ferrule in terms of height or/ and circumference presents better results than no ferrule at all or interrupted or less ferrule.

\section{References}

1. Magne $P$, Carvalho AO, Bruzi G, et al. Influence of no-ferrule and no-post buildup design on the fatigue resistance of endodontically treated molars restored with resin nanoceramic CAD/CAM crowns. Oper Dent 2014;39(6):595-602. DOI: 10.2341/13-004-L.

2. Eakle WS, Maxwell EH, Braly BV. Fractures of posterior teeth in adults. J Am Dent Assoc 1986;112(2):215-218. DOI: 10.14219/jada. archive.1986.0344.

3. Nagasiri R, Chitmongkolsuk S. Long-term survival of endodontically treated molars without crown coverage: a retrospective cohort study. J Prosthet Dent 2005;93(2):164-170. DOI: 10.1016/j. prosdent.2004.11.001.

4. Stankiewicz NR, Wilson PR. The ferrule effect: a literature review. Int Endod J. 2002;35(7):575-581. DOI: 10.1046/j.1365-2591.2002.00557.x.

5. Salehrabi R, Rotstein I. Endodontic treatment outcomes in a large patient population in the USA: an epidemiological study. J Endod 2004;30(12):846-850. DOI: 10.1097/01.don.0000145031.04236.ca.

6. Fokkinga WA, Kreulen CM, Vallittu PK, et al. A structured analysis of in vitro failure loads and failure modes of fiber, metal, and ceramic post-and-core systems. Int J Prosthodont 2004;17(4):476-482.

7. Goodacre CJ, Spolnik KJ. The prosthodontic management of endodontically treated teeth: a literature review. Part I. Success and failure data, treatment concepts. J Prosthodont 1994;3(4):243-250. DOI: 10.1111/j.1532-849x.1994.tb00162.x.

8. Schwartz RS, Robbins JW. Post placement and restoration of endodontically treated teeth: a literature review. J Endod 2004;30(5):289-301. DOI: 10.1097/00004770-200405000-00001.

9. Sorensen JA, Engelman MJ. Ferrule design and fracture resistance of endodontically treated teeth. J Prosthet Dent 1990;63(5):529-536. DOI: 10.1016/0022-3913(90)90070-s.

10. $\mathrm{Ng} \mathrm{CC}$, al-Bayat MI, Dumbrigue HB, et al. Effect of no ferrule on failure of teeth restored with bonded posts and cores. Gen Dent 2004;52(2):143-146.

11. Pissis P. Fabrication of a metal-free ceramic restoration utilizing the monobloc technique. Pract Periodontics Aesthet Dent 1995;7(5):8394.

12. Ramírez-Sebastià $A$, Bortolotto $T$, Roig $M$, et al. Composite vs ceramic computer-aided design/computer-assisted manufacturing crowns in endodontically treated teeth: analysis of marginal adaptation. Oper Dent 2013;38(6):663-673. DOI: 10.2341/12-208-L.

13. Pjetursson BE, Tan WC, Tan K, et al. A systematic review of the survival and complication rates of resin-bonded bridges after an observation period of at least 5 years. Clin Oral Implants Res 2008;19(2):131-141. DOI: 10.1111/j.1600-0501.2007.01527.x.

14. Howe DF, Denehy GE. Anterior fixed partial dentures utilizing the acid-etch technique and a cast metal framework. J Prosthet Dent 1977;37(1):28-31. DOI: 10.1016/0022-3913(77)90187-1.

15. Oesterle LJ, Cronin RJ, Ranly DM. Maxillary implants and the growing patient. Int J Oral Maxillofac Implants 1993;8(4):377-387.

16. Baldissara P, Llukacej A, Ciocca L, et al. Translucency of zirconia copings made with different CAD/CAM systems. J Prosthet Dent 2010;104(1):6-12. DOI: 10.1016/S0022-3913(10)60086-8. 
17. Zarone F, Di Mauro Ml, Ausiello $\mathrm{P}$, et al. Current status on lithium disilicate and zirconia: a narrative review. BMC Oral Health 2019;19(1):134. DOI: 10.1186/s12903-019-0838-x.

18. Magne P, Goldberg J, Edelhoff D, et al. Composite resin core buildups with and without post for the restoration of endodontically treated molars without ferrule. Oper Dent 2016;41(1):64-75. DOI: 10.2341/14258-L.

19. Forberger N, Göhring TN. Influence of the type of post and core on in vitro marginal continuity, fracture resistance, and fracture mode of lithia disilicate-based all-ceramic crowns. J Prosthet Dent 2008;100(4):264-273. DOI: 10.1016/S0022-3913(08)60205-X.

20. Shamseer $L$, Moher $D, C$ larke $M$, et al. Preferred reporting items for systematic review and meta-analysis protocols (PRISMA-P) 2015: elaboration and explanation. BMJ 2015;350:g7647. DOI: 10.1136/bmj. g7647.

21. Khabsa M, Elmagarmid A, llyas I, et al. Learning to identify relevant studies for systematic reviews using random forest and external information. Machine Learning 2016;102(3):465-482. DOI: 10.1007/ s10994-015-5535-7.

22. Sarkis-Onofre R, Skupien JA, Cenci MS, et al. The role of resin cement on bond strength of glass-fiber posts luted into root canals: a systematic review and meta-analysis of in vitro studies. Oper Dent 2014;39(1):E31-E44. DOI: 10.2341/13-070-LIT.

23. Goujat $A$, Abouelleil $H$, Colon $P$, et al. Marginal and internal fit of CAD-CAM inlay/onlay restorations: a systematic review of in vitro studies. J Prosthet Dent 2019;121(4):590-597.e3. DOI: 10.1016/j. prosdent.2018.06.006.

24. Naumann M, Preuss A, Rosentritt M. Effect of incomplete crown ferrules on load capacity of endodontically treated maxillary incisors restored with fiber posts, composite build-ups, and all-ceramic crowns: an in vitro evaluation after chewing simulation. Acta Odontol Scand 2006;64(1):31-36. DOI: 10.1080/00016350500331120.

25. Sherfudhin H, Hobeich J, Carvalho CA, et al. Effect of different ferrule designs on the fracture resistance and failure pattern of endodontically treated teeth restored with fiber posts and allceramic crowns. J Appl Oral Sci 2011;19(1):28-33. DOI: 10.1590/s167877572011000100007.

26. Fragou $T$, Tortopidis $D$, Kontonasaki $E$, et al. The effect of ferrule on the fracture mode of endodontically treated canines restored with fibre posts and metal-ceramic or all-ceramic crowns. J Dent 2012;40(4):276-285. DOI: 10.1016/j.jdent.2012.01.002.

27. Evangelinaki E, Tortopidis D, Kontonasaki E, et al. Effect of a crown ferrule on the fracture strength of endodontically treated canines restored with fiber posts and metal-ceramic or all-ceramic crowns. Int J Prosthodont 2013;26(4):384-387. DOI: 10.11607/ijp. 3409.

28. Valdivia A, Rodrigues MP, Bicalho AA, et al. Biomechanical effect of ferrule on incisors restored with a fiberglass post and lithium-disilicate ceramic crown after thermal cycling and fatigue loading. J Adhes Dent 2018;20(2):133-142. DOI: 10.3290/j.jad. a40305.

29. Einhorn M, DuVall N, Wajdowicz M, et al. Preparation ferrule design effect on endocrown failure resistance. J Prosthodont 2019;28(1):e237-e242. DOI: 10.1111/jopr.12671.

30. Zicari F, Van Meerbeek B, Scotti R, et al. Effect of ferrule and post placement on fracture resistance of endodontically treated teeth after fatigue loading. J Dent 2013;41(3):207-215. DOI: 10.1016/j. jdent.2012.10.004

31. Ma PS, Nicholls JI, Junge T, et al. Load fatigue of teeth with different ferrule lengths, restored with fiber posts, composite resin cores, and all-ceramic crowns. J Prosthet Dent 2009;102(4):229-234. DOI: 10.1016/S0022-3913(09)60159-1.

32. Silva-Sousa AC, Moris ICM, Barbosa AFS, et al. Effect of restorative treatment with endocrown and ferrule on the mechanical behavior of anterior endodontically treated teeth: An in vitro analysis. J Mechan Behav Biomed Mater 2020;112:104019. DOI: 10.1016/j. jmbbm.2020.104019.

33. Roscoe MG, Noritomi PY, Novais VR, et al. Influence of alveolar bone loss, post type, and ferrule presence on the biomechanical behavior of endodontically treated maxillary canines: strain measurement and stress distribution. J Prosthet Dent 2013;110(2):116-126. DOI: 10.1016/ S0022-3913(13)60350-9.

34. Ferrari M, Cagidiaco MC, Grandini S, et al. Post placement affects survival of endodontically treated premolars. J Dent Res 2007;86(8):729-734. DOI: 10.1177/154405910708600808.

35. Dikbas I, Tanalp J, Ozel E, et al. Evaluation of the effect of different ferrule designs on the fracture resistance of endodontically treated maxillary central incisors incorporating fiber posts, composite cores and crown restorations. J Contemp Dent Pract 2007;8(7):62-69. DOI: 10.5005/jcdp-8-7-62.

36. de Oliveira JA, Pereira JR, Lins do Valle A, et al. Fracture resistance of endodontically treated teeth with different heights of crown ferrule restored with prefabricated carbon fiber post and composite resin core by intermittent loading. Oral Surg Oral Med Oral Pathol Oral Radiol Endod 2008;106(5):e52-e57. DOI: 10.1016/j.tripleo.2008. 06.015 . 